\title{
Azimuthal anisotropy of long-range correlations at LHC energy in Monte Carlo model with string fusion
}

\author{
Vladimir Kovalenko ${ }^{1, a}$ \\ ${ }^{1}$ Saint Petersburg State University, Russia
}

\begin{abstract}
Long-range multiplicity correlations in intervals separated in pseudorapidity and azimuth are studied in the framework of string fusion approach. We applied a Monte Carlo model, in which the string configurations in the transverse plane and rapidity are simulating event-by-event. The string interaction is realized in the lattice string fusion approach with introduction of a grid in the transverse plane. We assumed that the azimuthal anisotropy of particle production is caused by parton energy loss traveling trough the media formed by clusters of fused strings : $\Delta p_{t} / \Delta x=-\alpha\left(p_{t} \sqrt{\eta}\right)^{2 / 3}$, where $\eta$ is a string density. In the cellular approach the Bresenham's line algorithm has been applied.It is obtained that in AA collisions, the parton energy loss seems to play considerable role, in particular, by providing large contribution to the correlation of mean transverse momentum with multiplicity. The developed approach provides non-zero values flows in $\mathrm{p}-\mathrm{Pb}$ collisions at LHC energies and produces the pattern similar to the one of the experimental di-hadron analysis.
\end{abstract}

\section{Introduction}

Currently ongoing studies of the collisions of hadrons and nuclei at high energies at RHIC and LHC colliders allow a detailed investigation of the properties of strongly interacting matter at extreme conditions. Recent experimental data indicate the presence of collective effects not only in the nucleus-nucleus collisions, but also in proton-nucleus, and even in the proton-proton interactions. So, in the CMS experiment at energy of $7 \mathrm{TeV}$ at high multiplicity so-called ridge-effect in pp collisions was first discovered, by observing the extended rapidity azimuthal structure of the two-particle correlation function [1]. Before it was only seen in the spectra of nucleus-nucleus collisions [2]. Similar structures later have also been found in the $\mathrm{p}-\mathrm{Pb}$ collisions at the LHC.

The present work is devoted to development of Monte Carlo approach model for the description of the azimuthal anisotropy of long-range correlations in soft and semi-hard region. Due to the fact that the multiple particle production processes are characterized by a rather low value of transverse momentum of particles, it leads to the impossibility of direct application of the pertrubative QCD and the need to develop semi-phenomenological approaches.

\footnotetext{
a e-mail: v.kovalenko@spbu.ru
} 
One of the models, which successfully describe particle production in soft region, is so-called quark-gluon string model. The model involves two-stage scenario of particles production: at the first stage extended in rapidity objects (quark-gluon strings, or colour tubes) are stretched between the partons of colliding hadrons, and they fragment into observable particles at the second stage. In order to study these extended in rapidity strings it was proposed to measure long-range correlations between observables from two rapidity windows, separated by a gap.

As the stirngs are finite objects in transverse plane, the overlapping of them and possible interactions can lead to non-trivial effects. Such interaction of the strings is considered in so-called string fusion model [5-7]. In particular, this model predicts the existence of non-zero long range correlations between multiplicity and mean transverse momentum (pt-n) and pt-pt correlations. The theoretical study of long-range correlations in the framework of string fusion model is carried out using Monte Carlo simulations [8-10]. The goal of this work is to include the mechanism of the azimuthal anisotropy [11-13] of the event-by-event particle production and to calculate the long-range correlation coefficient in the widows separated in rapidity and azimuth.

\section{The model}

The present model $[9,10]$ is based on partonic picture of nucleons interaction, accounting the energy and angular momentum conservation in the initial state of a nucleon. The nucleon is assumed to be composed of several set of pairs (quark-diquark and quark-antiquark), forming dipoles. The probability of dipoles interaction depends on the transverse coordinates of their ends $[14,15]$ with some effective coupling constant. Multiplicity and transverse momentum are calculated in the approach of colour strings, stretched between projectile and target partons, taking into account their finite rapidity width and interaction in transverse plane - string fusion $[16,17]$. The main effect of the fusion of strings is modification of the mean transverse momentum and multiplicity, coming from a cluster of overlapping strings:

$$
\langle\mu\rangle_{k}=\mu_{0} \sqrt{k} \frac{S_{k}}{\sigma_{0}}, \quad\left\langle p_{t}^{2}\right\rangle_{k}=p_{0}^{2} \sqrt{k}, \quad\left\langle p_{t}\right\rangle_{k}=p_{0} \sqrt[4]{k},
$$

where $S_{k}$ - area, where k strings are overlapping, $\sigma_{0}$ - single string transverse area, $\mu_{0}$ and $p_{0}-$ mean multiplicity and transverse momentum from one string. A string (or cluster of overlapping strings) fragments independently in any rapidity interval according to Poisson distribution. The model parameters are constrained from the p-p data on total inelastic cross section and multiplicity and multiplicity in minimum bias $\mathrm{p}-\mathrm{Pb}$ and $\mathrm{Pb}-\mathrm{Pb}$ collisions [18].

For the azimuthal anisotropy of particle production we assumed that it is caused by parton energy loss traveling trough the media formed by clusters of fused strings [11-13]:

$$
\Delta p_{t} / \Delta x=-\alpha\left(p_{t} \sqrt{\eta}\right)^{2 / 3}
$$

where $\eta$ is a string density. In the cellular approach the Bresenham's line algorithm [19] has been applied. 


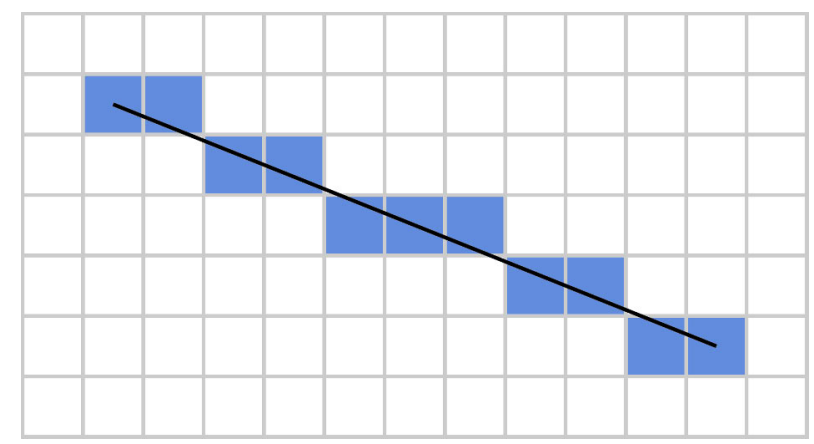

Figure 1. Illustration of Bresenham's line algorithm applied to calculation of the loss of transverse momentum.

\section{Results}

First, we calculated the correlation between transverse momentum and multiplicity in $\mathrm{Pb}-\mathrm{Pb}$ collisions at LHC energy. Previously we found [20] that it is not possible to consistently describe $\left\langle p_{T}\right\rangle_{N_{\mathrm{ch}}}-N_{\mathrm{ch}}$ in $\mathrm{pp}, \mathrm{p}-\mathrm{Pb}$ and $\mathrm{Pb}-\mathrm{Pb}$ collisions without considering any energy loss in case of AA interaction. In the present work, with introducing of the parton energy loss effect, and by varying of the parameter $\alpha$, which characterize it's strength, one allowed to describe the $\left\langle p_{T}\right\rangle_{N_{\mathrm{ch}}}-N_{\mathrm{ch}}$ correlation (see figure 2).

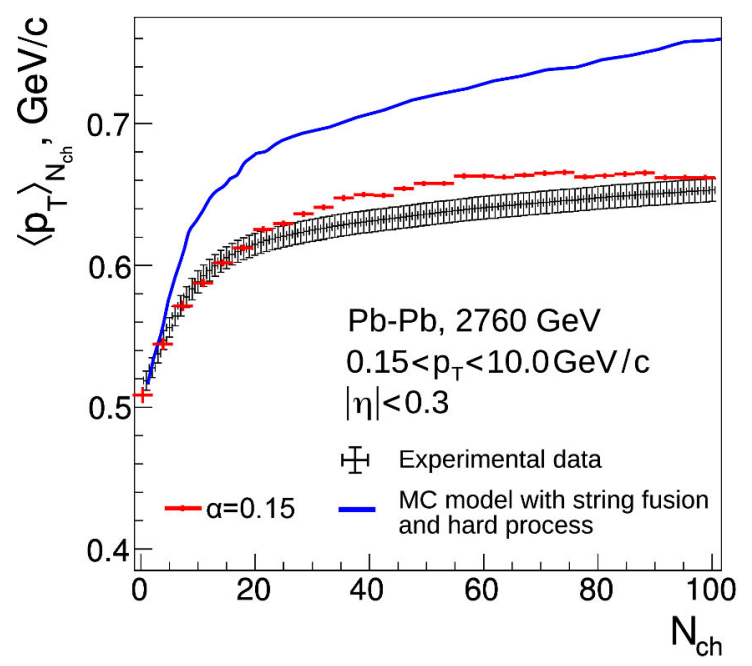

Figure 2. pt-n correlations in $\mathrm{Pb}-\mathrm{Pb}$ collisions with (red dots) and without (blue line) momentum loss, compared to the experimental data [21]

The long range correlations between observables in two windows separated in rapidity and azimuth (backward $B$ and forward $F$ ) [22] are numerically characterized by correlation coefficient, which is defined as: 


$$
b=\frac{\langle B F\rangle-\langle B\rangle\langle F\rangle}{\sqrt{\operatorname{Var} B} \sqrt{\operatorname{Var} F}} .
$$

Here for observables in each window $(B, F)$ we used $N_{\mathrm{ch}}$ - the number of charged particles in the window. The windows configuration is showed at the right panel of figure 3 .

The azimuthal structure of the multiplicity correlations has been calculated for $\mathrm{p}-\mathrm{Pb}$ collisions at $\sqrt{s}=5.02 \mathrm{TeV}$, centrality $0-20 \%$. The centrality of events were defined according to multiplicity in a forward rapidity region [23].

The results show that the developed approach provides non-trivial azimuthal structure of longrange correlations, including the presence of near-side and away-side ridges. Note that it implies the non-zero values flows in $\mathrm{p}-\mathrm{Pb}$ collisions at LHC energies. Overall the obtained pattern is similar to the picture obtained by di-hadron analysis of the experimental data [24].
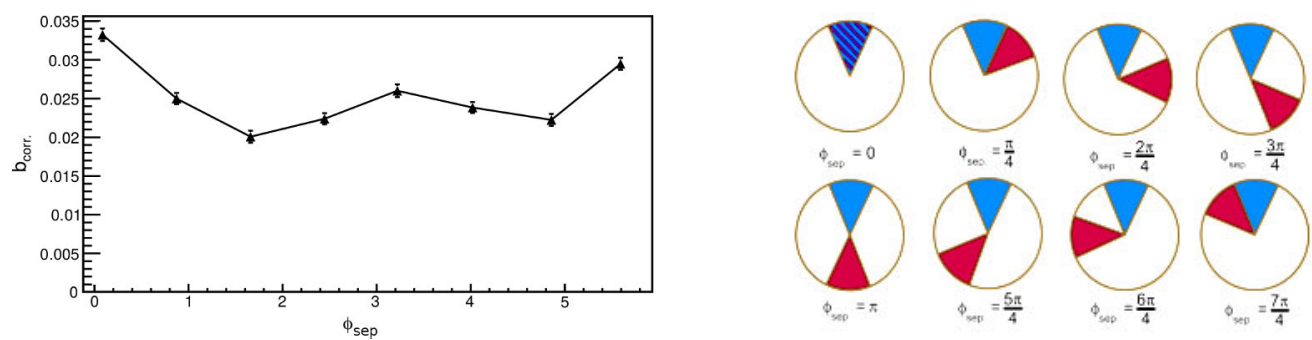

Figure 3. Dependence of the multiplicity correlation coefficient on the azimuthal separation of windows. The right panel shows the windows configurations.

\section{Summary and outlook}

Long-range (forward-backward) correlations are studied in the framework of string fusion approach. We developed and applied a Monte Carlo model in which the string configurations in the transverse plane and rapidity are simulating event- by-event. We assumed that the azimuthal anisotropy of particle production is caused by parton energy loss traveling trough the media formed by clusters of fused strings and implemented the mis mechanism using cellular string fusion model This developed approach provides non-trivial azimuthal topology of long-range correlations $\mathrm{p}-\mathrm{Pb}$ collisions at LHC energies. In AA collisions, the parton energy loss seems to play considerable role, in particular, by providing large contribution to the mean transverse momentum and its correlation with multiplicity. Further developments will include the modelling of short-range peak, $\mathrm{pp}, \mathrm{Pb}-\mathrm{Pb}$ colliding systems, comparison of several scenarios of string fusion, studies the azimuthal structure of different types of long-range correlations: n-n, pt-pt, pt-n [25].

\section{Acknowledgements}

The author acknowledges Saint-Petersburg State University for the research grants 11.38.197.2014 and 11.42.995.2016. 


\section{References}

[1] V. Khachatryan et al. (CMS Collaboration), JHEP 1009, 091 (2010).

[2] B. Alver et al. (PHOBOS Collaboration), Phys. Rev. Lett. 104, 062301 (2010).

[3] A. Capella, U.P. Sukhatme, C.I. Tan, Van J. Tran Thanh, Phys. Rep. 236, 225-329 (1994).

[4] H. J. Drescher, M. Hladik, S. Ostapchenko, T. Pierog, K. Werner, Phys. Rept. 350, 93 (2001).

[5] N.S. Amelin, N. Armesto, M.A. Braun, E.G. Ferreiro, C. Pajares, Phys. Rev. Lett. 73, 2813 (1994)

[6] M. Braun, F. del Moral, C. Pajares. Eur. Phys. J. C 21, 557 (2001)

[7] M. A. Braun, J. Dias de Deus, A. S. Hirsch, C. Pajares, R. P. Scharenberg and B. K. Srivastava, Phys. Rept. 599, 1 (2015).

[8] V.V. Vechernin, R.S. Kolevatov, Phys. Atom. Nucl. 70, 1797 (2007)

[9] V. N. Kovalenko, Phys. Atom. Nucl. 76, 1189 (2013), arXiv:1211.6209 [hep-ph]

[10] V. Kovalenko, V. Vechernin, PoS (Baldin ISHEPP XXI) 077 (2012), arXiv:1212.2590 [nucl-th]

[11] I. Bautista, L. Cunqueiro, J. D. de Deus and C. Pajares, J. Phys. G 37, 015103 (2010).

[12] M. A. Braun, C. Pajares and V. V. Vechernin, Nucl. Phys. A 906, 14 (2013).

[13] M. A. Braun, C. Pajares and V. V. Vechernin, Eur. Phys. J. A 51, no.4, 44 (2015).

[14] C. Flensburg, G. Gustafson, and L. Lonnblad, Eur. Phys. J. C 60, 233 (2009), arXiv:0807.0325

[15] G. Gustafson, Acta Phys. Polon. B 40, 1981 (2009) arXiv: 0905.2492.

[16] M.A. Braun, C. Pajares, and V.V. Vechernin, Phys. Let. B 493, 54 (2000).

[17] M. Braun, R. Kolevatov, C. Pajares, and V. Vechernin, Eur. Phys. J. C 32, 535 (2004)

[18] V. Kovalenko, PoS (QFTHEP 2013) 052 (2013).

[19] J. E. Bresenham, IBM Syst. J 4, no. 1, 25-30 (1965).

[20] V. Kovalenko, V. Vechernin, presentation at Quark Matter 2014, Darmstadt (2014), https://indico.cern.ch/event/219436/contributions/1523280/.

[21] Abelev B., et al. (ALICE Collaboration), Phys. Lett. B. 727, 371 (2013).

[22] J. Adam et al. (ALICE Collaboration), JHEP 1505097 (2015), arXiv:1502.00230 [nucl-ex].

[23] B. Abelev et al. (ALICE Collaboration), Phys. Rev. C 88, no.4, 044909, (2013), arXiv:1301.4361 [nucl-ex].

[24] L. Milano, J. Phys. Conf. Ser. 509, 012105 (2014), arXiv:1309.3457.

[25] B. Alessandro et al. (ALICE Collaboration), ALICE: Physics Performance Report, Vol. II, J. Phys. G 32, 1295 (Section 6.5.15: Long-range correlations, p. 1749) (2006). 